\title{
LAS ESCALAS DE LO SOCIAL
}

\section{The scales of the social}

\author{
Margarita Barañano* \\ * Universidad Complutense de Madrid \\ mbaranan@ccee.ucm.es
}

\section{Palabras clave \\ Escala \\ Multiescalaridad \\ Salto de escalas \\ Dislocaciones \\ escalares \\ Políticas de escala \\ Des/re-articulación \\ escalar}

\section{Keywords}

Scale

Multi-scale

Jumping of scale

Scale's dislocations

Politics of scale

Scalar des/re-

articulation

\begin{abstract}
Resumen
Si bien el estudio de las transformaciones de lo social a partir de la noción de escala no es central en la obra de Pérez-Agote, cabe encontrar en ella análisis que resultan de utilidad al respecto. En primer lugar, se apoya en una consideración multiescalar y multidimensional de la vida social, que tiene en cuenta no sólo los aspectos socio-económicos o su delimitación político-administrativa sino también su fundamentación territorial, simbólica e identitaria. Esta aproximación le permite examinar algunas de las dislocaciones más debatidas de la modernidad, que la mundialización agudiza, como la derivada de la separación creciente entre la escala territorial, preferentemente local, en la que se despliegan nuestras vidas, de un lado, y aquellas otras escalas, cada vez más distantes, en las que se gestan muchos de los procesos que afectan de un modo central a dichas vidas. También facilita tener en cuenta la atención a distintas escalas emergentes, como la subestatal. Pérez-Agote ofrece, además, valiosos análisis para una comprensión multiescalar alejada de su equiparación con una suerte de jerarquización lineal y constante entre las distintas escalas, así como para comprender el "salto de escalas" y las "política de escala". Por último, los debates sobre el estatuto científico de la noción de escala se asemejan a los referidos a conceptos estratégicos en su obra, como el de nación.
\end{abstract}

\section{Abstract}

While studying social transformations from the notion of scale is not central to the work of Pérez-Agote, there are multiple insights in his work that are useful in this regard. First, it is based on a multi-scale and multidimensional consideration of social life, which takes into account not only its socio-economics aspects or its political-administrative territorial demarcation but also its symbolic foundation and its relevance to collective identities. His approach allows to examine some of the most debated dislocations of modernity, that globalization has deepened, as the dilemma between the territorial scale where our lives unfold and those other distant scales where the logics that shaped these lives are produced. This perspective also facilitates the consideration of different emerging scales, as the regional or the local. Perez-Agote also offers valuable analysis for the understanding of the scalar structuration of social life away from its comparison with a kind of linear and constant hierarchy between the different scales, and to understand the "jump of scale" and the "politics of scale ". Finally, discussions on the scientific status of the concept of scale are similar to those related to strategic concepts in his work, like the nation.

Barañano, M., 2015, "Las escalas de lo social", en Papeles del CEIC, vol. 2015/3, no 137, CEIC (Centro de Estudios sobre la Identidad Colectiva), Universidad del País Vasco, http://dx.doi.org/10.1387/pceic.14881 


\section{INTRODUCCIÓN ${ }^{1}$}

Conviene comenzar este artículo con una aclaración: la tesis sostenida en el mismo no es tanto que la noción de escala, de relevancia creciente en las ciencias sociales (Herod, 2011; Herod y Wright, 2002a; Barañano, 2005) sea central en la obra de Alfonso Pérez-Agote, ni siquiera que se trate de un concepto profusamente citado en su obra. Tampoco, seguramente, cabe considerar que el análisis de la dimensión espacial de los fenómenos a los que ha dedicado más atención sea uno de los ejes centrales de su trabajo.

Lo que se quiere plantear es que la obra de Pérez-Agote ofrece múltiples aportaciones de interés en relación con el análisis de las reconfiguraciones de las identidades colectivas, o de la vida social y cultural, en diferentes escalas. La primera de ellas es que su trabajo se abre a la reconfiguración multiescalar de lo social. Esto no equivale a decir que no tenga en cuenta, por ejemplo, la centralidad de la escala del Estado-nación en la primera sociedad moderna. Lo que sucede es que este reconocimiento convive en su obra con una comprensión de esta realidad como plural, objeto de diferentes interpretaciones sociales en distintos contextos. Este será el caso, por ejemplo, de la escala de la nación, delimitada de manera muy diferente por el Estado o desde los nacionalismos periféricos. A lo que habría que añadir la panoplia de instituciones intermedias, referidas a otras escalas, que habrían nacido o adoptado nuevas versiones en las emergentes sociedades modernas.

Se afirma también en este trabajo que este autor consigue abrirse a esta diversidad de escalas apoyándose, entre otras interpretaciones, en una lectura no dicotómica de la distinción durkheimiana entre la solidaridad mecánica y la orgánica, así como en una comprensión del Estado y de la sociedad moderna nacional inspirada en Weber. Esta comprensión, siguiendo, también a Nisbet, lejos de evacuar la comunidad, o las identidades colectivas, las integra dentro de la nueva realidad social, como una parte central de la misma. Esto es, las instituciones -

\footnotetext{
${ }^{1}$ Este artículo se ha redactado durante mi estancia de investigación como Visiting Researcher en el Departamento de Sociología de la Universidad de Cambridge, desde el 1 de julio al 30 de septiembre de 2015. Agradezco muy sinceramente a esta institución, y a las personas que la integran, especialmente a la Profesora Jacqueline Scott, la amable acogida y la oportunidad que se me ha brindado de trabajar en un contexto tan estimulante. Extiendo el agradecimiento al GRESCO, del Instituto Complutense para el Estudio de las Transformaciones Sociales Contemporáneas (TRANSOC) y del Instituto de Investigaciones Feministas de esta misma Universidad.
} 
¿escalas? - primarias e intermedias, cargadas de identidad y de sentido, no habrían dejado de ser relevantes, si bien su significación habría cambiado en el nuevo entorno social, como volverá a acaecer en el contexto de la mundialización. Es más, la centralidad de cada una de estas instituciones intermedias, su configuración concreta, y las modalidades de des/re-articulación entre ellas, lejos de responder a un patrón inalterable, presentarían una gran variabilidad e inestabilidad, según, entre otros factores, a las prácticas sociales y las estructuras de plausibilidad en juego (Pérez-Agote, Tejerina y Barañano, eds., 2010: 210), relativas a tiempos y contextos específicos.

Además, Pérez-Agote ofrece una temprana interpretación de algunas de las que se han considerado como dislocaciones constitutivas de lo social en la modernidad. Entre ellas, cabe citar la recogida en la frase, reiterada en diferentes momentos de su obra, conforme a la cual

"la sociedad local sigue significando para el individuo un lugar donde todo lo que pasa le afecta pero ya no es el lugar donde pasa todo lo que le afecta, pues hay cosas que le afectan que vienen de lejos" (Pérez-Agote, 2005: 3).

En sus propios términos, "la sociedad local comienza a estar "exocentrada" (Pérez-Agote, 1996: 59) no ya en la era global, sino mucho antes, una vez que la escala correspondiente al Estado-nación se convierte en hegemónica. Algo a lo que se referirá también un autor como Jameson (1991), con su alusión a la creciente separación entre el espacio en el que se despliegan nuestras vidas y aquel en el que se conforman las condiciones estructurales de su producción y de su posibilidad. Estas últimas resultarian, según Jameson, cada vez más incognoscibles e irrepresentables para los actores sociales.

Los trabajos de Alfonso Pérez-Agote ofrecen así análisis de utilidad para aproximarse a la compleja des/re-configuración de las relaciones entre dichas escalas y sus distintas dimensiones, que, en algunos casos, suponen su interpenetración $y$, en otros, una serie de tensiones o dislocaciones. Entra en esta última situación también la que constata, por ejemplo, en el caso del nacionalismo vasco, entre el sentimiento de pertenencia a una comunidad de base territorial, de un lado, y la delimitación político-administrativa de la misma, de otro, o, expresado en otros términos, la configurada por la afirmación del nacionalismo periférico vasco, de una parte, y el grado de objetivación política conseguida por el mismo, de otra, al carecer de un Estado propio. 
Su obra supone también una importante y temprana contribución a la tarea de desvelar el nacionalismo metodológico implícito en el nacimiento y la institucionalización de la sociedad moderna equiparada en muchas de las interpretaciones con la sociedad nacional一, y de la propia sociología, que encontró desde sus inicios en el Estado un contenedor central de dicha sociedad y de sus límites. Este análisis ha constituido un eje central de la reflexión de importantes analistas sobre la globalización (Sassen, 2008; Castells, 1997, 2006), el transnacionalismo (Wimmer y Glick Schiller, 2002), o bien respecto del nuevo modelo social (Beck, 1996; o Giddens, 1993).

Como se ha señalado, Pérez-Agote consigue realizar estas aportaciones abriéndose a la consideración de las múltiples escalas de lo social, y, en particular, al ascenso de nuevas escalas emergentes y a su reposicionamiento en el mundo actual. Este es el caso, muy especialmente, de la escala subestatal, correspondiente, de manera singular, a las naciones sin Estado, pero también de otras, como el barrio $u$ otras esferas locales.

En su obra se recogen, además, análisis de procesos en una dirección confluyente con los que en la literatura de las escalas espaciales han recibido una notable atención, como la cuestión de "las políticas de escala" (Cox, 1998a, 1998b; Brenner, 2001), "del salto de escalas" (Swyngedouw, 1992, 1997) u otras. Todo ello, por último, suscita la pregunta por la propia definición de escala, que ha conocido hasta ahora múltiples versiones, así como por el estatuto teórico de esta noción (Herod, 2011; Herod y Wright, 2002a; Jones, 1998).

En los siguientes apartados se abordan los aspectos más arriba citados, siguiendo un enfoque, en su inicio, más historicista que sistemático, por entenderlo afin al propio tratamiento por Alfonso Pérez-Agote de las nociones que aquí se consideran. Se finaliza con unas muy breves consideraciones finales, de doble dirección, tanto por lo que hace a la justificación del examen de la obra del autor a la luz de la noción de escala como, en sentido inverso, por lo que se refiere a la contribución de su trabajo para aproximarnos a los debates relativos al interés y al estatuto de esta noción. 


\section{LA ESCALA DEL ESTADO-NACIÓN Y LAS ESCALAS "INTERMEDIAS"}

Alfonso Pérez-Agote, ya en su obra de 1989, incide en el desvelamiento de lo que luego se ha denominado el "nacionalismo metodológico" (Beck, 1998, 2006), poniendo de manifiesto cómo "el origen, la institucionalización y el desarrollo de la sociología -en una palabra, su historia- están ligados indefectiblemente al auge del Estado-nación occidental" (1989: 15). Por esta razón, la sociología se habría interesado más por la sociedad que por lo social, entendida como lo social determinado por el Estado.

El Estado "corta y divide la realidad social indeterminada, $y$, en cierto modo, difusa, en realidades sociales totales objetivadas" (Pérez-Agote, 1989: 18) delimitando de este modo la noción de sociedad. Así, “las sociedades nacionales de los siglos XIX y XX han constituido el correlato empírico de la noción de sociedad" (Ibid.: 45). Estas sociedades, determinadas por sus respectivos Estados, habrian ofrecido a la sociología naciente "una hipótesis de seguridad, la mayoría de las veces implícita", (Íbidem) sobre la que fundar sus apreciaciones.

Ahora bien, para Pérez-Agote la definición de las sociedades nacionales no se agota en la delimitación puramente territorial de sus fronteras por los Estados. Por el contrario, presta una gran atención a su configuración multidimensional, incluyendo tanto los aspectos físicos o materiales como los simbólicos y subjetivos, refiriendo estos últimos, entre otras cuestiones, al sentimiento de pertenencia a la comunidad nacional. Pérez-Agote hace descansar esta aproximación a la complejidad de estas sociedades y del Estado en una determinada interpretación de la contribución de Weber en esta materia, quien habría subrayado la multidimensionalidad de estas realidades, ya desde la primera modernidad. Esta comprensión de la sociedad moderna y del Estado, inspirada en Weber, y que Pérez-Agote aborda en distintos trabajos (Pérez-agote, 1989: 16-18; 2008: 58-61), subraya la dimensión territorial de la escala que configuran, y también su conformación en tanto que comunidad nacional. Esto es, en tanto que "lugar simbólico" y "comunidad segregada" (Pérez-Agote, 2008: 65), no sólo por sus fronteras sino también por el sentimiento de pertenencia a la misma.

El reconocimiento de la pluralidad de dimensiones implicadas en la conformación de los Estados-nación, y de las sociedades emergentes, así como de las dislocaciones posibles entre estas dimensiones, abren la 
puerta a la consideración de las escalas intermedias en la comprensión de Pérez-Agote sobre la conformación de lo social en las sociedades modernas. En algunos casos, se da una coincidencia entre "elemento objetivo y el subjetivo", entre "la extensión territorial del Estado y la extensión de la conciencia de pertenencia nacional" (Pérez-Agote, 2008: 58 ) en el que se sustenta esta forma organizativa, como sucede, por ejemplo, con el nacionalismo central. Pero, como añade, también "puede no existir tal coincidencia, como en el caso de que en un territorio más pequeño que el estatal se dé un sentimiento de pertenencia excluyente del sentimiento de dimensión estatal" (PérezAgote, 2008: 59). En esta situación, la escala del Estado-nación, aun siendo hegemónica en la objetivación de la sociedad, coexiste, como mínimo, con la correspondiente a la del espacio subestatal en las prácticas y los discursos de los actores, y en el conjunto de la configuración de la vida social y cultural. La nación, además, deviene una escala en disputa, poniendo de manifiesto su carácter contextual y relacional y la variabilidad de su significado y contenidos, algo aplicable a la propia noción de escala.

Es cierto, de otro lado, que, por lo que hace a otras escalas inferiores, Alfonso Pérez-Agote nos recuerda cómo ya Durkheim se refirió a la "pérdida de importancia social de las agrupaciones territoriales y los sentimientos de pertenencia que suscitan" (Durkheim en Pérez-Agote, 1989: 30), al hecho de que los "lazos que nos unen a un municipio, a un departamento, devienen más frágiles y débiles" (Dukheim en PérezAgote, 1989: 30), de modo que el patriotismo de campanario ha llegado a constituir un arcaísmo que no cabe restaurar" (Dukheim en PérezAgote, 1989: 31).

A partir de esta comprensión, se prefigura ya una de las dislocaciones de la modernidad, que se profundizará con la mundialización, a la que Durkheim atribuye una de las fuentes de la anomía y que Pérez-Agote relaciona con el carácter crecientemente opaco del mundo para los actores sociales. Dicha dislocación consiste en que, si bien la vida se sigue desenvolviendo en espacios locales, lo que nos afecta se produce cada vez menos en su interior, respondiendo, en muchos casos, a lógicas cuyos centros de decisión están fuera. Como ya se ha mencionado, este planteamiento se asemeja al establecido por Jameson (1991) entre el espacio de la experiencia vivida, de una parte, y el hiperespacio en el que se producen las condiciones de dicha experiencia, de otra. En su libro de 
2008, Pérez-Agote complejiza esta formulación, distinguiendo entre este tipo de dislocación, que atribuye a la globalización económica, y la derivada de la globalización cultural, conforme a la cual el individuo "alcanza a conocer ámbitos del mundo que no le afectan en su vida" (Pérez-Agote, 2008: 34). Proporciona así reflexiones valiosas para comprender estas tensiones contemporáneas no sólo en relación con los desplazamientos entre unas escalas y otras, sino también por lo que hace a las dinámicas de las distintas dimensiones de cada una de ellas.

Ahora bien, a renglón seguido, y de acuerdo también con una determinada comprensión de la obra de Durkheim, Pérez-Agote recuerda que, incluso en la etapa de predominio del "unitario territorio estatal", se hace necesaria una nueva "vertebración de la sociedad que sustituya a la antigua" (Pérez-Agote, 1989: 31), apoyada en otras agrupaciones, correspondientes a otras escalas. Como expone Durkheim en la cita recogida en la obra de Pérez-Agote:

\footnotetext{
"Una nación no puede mantenerse como no se intercale entre el Estado y los particulares toda una serie de grupos secundarios que se encuentren lo bastante próximos de los individuos como para atraerlos fuertemente a su esfera de acción y de conducirlos así en el torrente general de la vida social" (Durkheim en Pérez-Agote, 1989: 31).
}

Una proximidad que cabe entender como emocional y simbólica, de sentido compartido, y que, además, en algunos casos, es también espacial y territorial. Como señala nuestro autor, Durkheim encuentra este "nivel" intermedio, esta agrupación social secundaria, en la corporación profesional, capaz de generar, a su parecer, integración y sentido de pertenencia. Y como añade: "no importa tanto aquí el tan criticado (...) carácter corporativo del Estado durkheimiano cuanto los niveles sustanciales, en términos de escala" (Pérez-Agote, 1989: 31). En definitiva, a partir de una interpretación no dicotómica de la distinción de Durkheim entre los tipos de solidaridad, según la cual la nueva "solidaridad orgánica" no supone la aniquilación de la "mecánica" ni tampoco la de todas las formas de comunidad y de sentido, "sustituidas por el contrato y el individuo aislado" (íbidem), Pérez-Agote subraya cómo, además de "la escala territorial del Estado-nación, destaca la esfera correspondiente a la "articulación de niveles, asociaciones e instituciones que ofrecen la mediación entre el individuo y el Estado" (Ibid.: 33) y que son capaces de generar sentimientos de pertenencia.

En la comprensión de Pérez-Agote, los sentimientos comunitarios de pertenencia y las identidades colectivas, por tanto, ni desaparecen ni se 
agotan en los marcos de lo Estatal-nacional o de "Ia gran nación". Ello concuerda con su referencia a que la comunidad y la identidad nunca se acabaron de ir, aunque adoptaran entonces versiones distintas. Esta perspectiva facilita su comprensión de la relevancia de otras escalas, como la subestatal, la local u otras, vinculadas a demarcaciones territoriales o comunitarias; del carácter contingente y relativo de todas ellas, como es el caso de la nacional pero también de la local o subestatal, que no siempre son claves, pero que tampoco están condenadas a convertirse en "fantasmagóricas", como señalará algún autor (Giddens, 1993); de la pluralidad de escalas en juego; del componente no sólo territorial sino también simbólico de las escalas, como espacios narrativos y de identificación (Herod y Wright, 2002b) que pueden sustentar comunidades de vida y de acción; o, en fin, de su configuración relacional, en tanto que red de relaciones sociales, de acuerdo con la definición constructivista de la escala ofrecida por Cox (1998a,1998b).

\section{LA MUNDIALIZACIÓN Y LA DES/RE-ARTICULACIÓN DE NUEVAS Y "VIEJAS" ESCALAS}

Según el planteamiento de Pérez-Agote, en las décadas recientes, de la mano de la mundialización, asistimos no tanto un desvanecimiento de la escala del Estado-nación, o a una desaparición de las escalas intermedias, cuanto a diferentes des/re-articulaciones de las relaciones entre ellas $y$, en algunos casos, a una redefinición de sus respectivos significados y delimitaciones geográficas. En sus palabras:

"la sociedad nacional como totalidad precisamente articulada y organizada, objetivada territorialmente, se desorganiza, se desarticula, dando lugar a una multiplicidad creciente de movimientos, conflictos y tensiones, mientras que, desde fuera, se le imponen y superponen, de manera objetiva, otras entidades - [¿escalas?]— sociales" (Pérez-Agote, 1989: 46).

El proceso de "progresiva mundialización" y la nueva sociedad global -a la que hay ya una alusión temprana del autor (Ibid.: 36)-, se habría acompañado de un fortalecimiento en Europa de los nacionalismos periféricos, así como de una proliferación de nuevos conflictos, algunos de los cuales tendrían una importante dimensión espacial, como los movimientos sociales urbanos, ecologistas, o regionalistas. Lo que estaría casi siempre en juego en estos conflictos, según el autor, sería la identidad colectiva y la comunidad, caracterización esta que se traslada fácilmente al ámbito de la escala espacial. No se trataría tampoco de un reemplazo de la centralidad de la escala del Estado-nación por una rejerarquización de las escalas conforme a un nuevo orden, igualmente 
invariable y estable, como si se estuviéramos ante un acoplamiento prefijado de las mismas, de acuerdo con la extensión relativa de cada una de ellas, como en el caso de las muñecas rusas. Por el contrario, lo interesante, también en este caso, sería estudiar el cómo de la cristalización de estas escalas y de sus des/re-articulaciones, teniendo en cuenta la diversidad de modalidades de estos procesos, objetivo que el autor reitera en su investigación sobre los nacionalismos periféricos. Las contribuciones de Pérez-Agote respecto del carácter situado de las identidades colectivas, y la variación de las escalas que se destacan en la definición de las mismas, según el contexto en el que tienen lugar las interacciones, resultan también de utilidad para aproximarse a la variabilidad de lo que, siguiendo a Herod, cabe denominar como las nuevas "estructuraciones escalares" (Herod, 2011: 28). El hecho de que en la interacción cotidiana se subraye, sobre todo, la identidad nacional compartida en el encuentro de dos o más personas frente a terceros de otras nacionalidades; de que, por el contrario, se priorice con frecuencia la identidad local, correspondiente, por ejemplo, a la condición de costeño o de serrano, cuando la interacción no incluye a extranjeros; o de que una persona de Brasil y otra de Ecuador sean identificadas como "latinas" frente a otras procedentes, por ejemplo, de China, como ha puesto de manifiesto el autor en su estudio sobre los barrios multiculturales (Pérez-Agote et al., 2010), ofrece un valioso ejemplo de los posibles reposicionamientos de las escalas en distintos contextos. En estos procesos jugarían un papel relevante no sólo los factores económicos o estrictamente territoriales sino también la "explosión" de los sentimientos de pertenencia y de comunidad.

Resulta interesante, en esta dirección, considerar los análisis de PérezAgote que, si bien no aluden directamente a las escalas de lo social, ofrecen interesantes herramientas para avanzar en su conocimiento. Así sucede, entre otros aspectos, con el análisis de las transformaciones de la forma Estados-nación, que, lejos de declinar, se expande de manera notable en la era global. También se constata en relación con uno de los cambios de la nación, y, por ende, del nacionalismo, que más atañe a su dimensión espacial, y que tiene que ver con el ascenso del transnacionalismo. Se trata del desbordamiento de las fronteras de las naciones por parte de flujos que antes desplegaban su actividad, sobre todo, en el interior de estas, y que, ahora, cada vez más, habitan una nueva espacialidad transnacional. Otro cambio en esta misma dirección tendría que ver con las nuevas posibilidades de "estiramiento", más allá de sus fronteras espaciales iniciales, de las redes de sentido y de identidad, como constata en el caso de una escala subestatal, la del nacionalismo periférico vasco, evidenciado por la investigación de Pérez-Agote sobre la comunidad vasca de Nevada. Proceso este que 
ilumina de manera muy ilustrativa el fenómeno de re-articulación de escalas llamado por Anderson (1993) "nacionalismo a distancia". En este marco, además, no es sólo la nación la que se modifica, sino que también lo hacen las restantes escalas, desde las correspondientes al ámbito de los barrios o los espacios locales como las referidas a los espacios subestatales o incluso al propio ámbito global.

Este es el caso, por ejemplo, de los barrios multiculturales de los cascos históricos, convertidos algunos de ellos en "refugios" (Pérez-Agote et al., 2010: 208) decisivos para muchas de las nuevas poblaciones migrantes extranjeras, al menos, durante una primera etapa de incorporación a la vida social en el contexto de llegada. Algo semejante parece suceder en algunos de los barrios periféricos de vivienda social que han recibido o están recibiendo a estos transmigrantes de primera generación, conforme se pone de manifiesto en una reciente investigación del autor. La variabilidad de formas de este tipo de escala se evidencia, además, en el hecho de que ya en etapas previas, y en contextos muy distintos, los barrios constituyeron también una escala decisiva en la conformación de la identidad colectiva y del sentimiento de pertenencia, como PérezAgote estudia en relación con los procesos de socialización en el nacionalismo vasco durante la etapa franquista. Según su investigación, en el marco de una esfera pública negadora de la existencia de esta nación periférica, la socialización en el sentimiento de pertenencia a la misma se recondujo hacia los ámbitos preferentemente localistas y privados, más difíciles de controlar por el Estado, como la cuadrilla, las parroquias o el barrio, que vieron así reforzada su posición en estos territorios.

Encontramos aquí otra contribución de la obra de Pérez-Agote a la comprensión de cómo la configuración de las escalas no tiene lugar conforme a un proceso universal, uniforme o invariable sino que, por el contrario, es un producto inestable e incierto de las estructuras de plausibilidad, de una parte, y de las prácticas y sentidos compartidos de los actores sociales, de otra, presentes en un contexto determinado. A lo que hay que añadir que esto no sucede sólo en relación con las escalas más "micro", como las recientemente citadas, sino que las nuevas condiciones características de la mundialización hacen que también se produzcan procesos en esta dirección en la escala global. La nueva ciudadanía que, siguiendo a Albrow (1997), denomina performativa global, posibilitada tanto por el sentimiento de pertenencia a la globalidad como por la comunicación mundialmente expandida, apuntaría en esta dirección.

Conviene dar cuenta, por último, de tres procesos importantes referidos a la des/re-constitución multiescalar de la vida social actual, respecto de 
los que la obra de Pérez-Agote proporciona reflexiones de interés. En primer lugar, la penetración de procesos gestados en escalas de mayor extensión en la producción de dinámicas sociales presentes en espacios correspondientes a escalas geográfica inferiores, como los barrios o los enclaves locales de los llamados negocios étnicos, ofrece un ejemplo de interés del significado de la multiescalaridad. En segundo lugar, las referencias de algunas de las escalas subestatales de la Unión Europea, definidas, sobre todo, por nacionalismos periféricos, respecto del espacio supranacional de la Unión, y sus expectativas, en algunos casos, de obtención de un mayor reconocimiento en este último ámbito que en el de sus respectivos Estados-nación, es un fenómeno analizado por Pérez-Agote que resulta útil para ilustrar el concepto de "salto de escalas" (Swyngedouw, 1992 y 1997, Cox, 1998a y 1998b). En tercer lugar, hay que destacar también que las posibilidades de jugar con el salto de escalas no es algo ajeno al poder de los actores y las estructuras en juego, en la dirección del denominado "poder de escala" (Brenner,1997; Brenner, 2001; Cox, 1998a, 1998b; Delaney y Leitner, 1997; Smith, 1990).

\section{REFLEXIONES FINALES}

Como señaló Lefebure, "la cuestión de la escala y el nivel implica una multiplicidad de escalas y de niveles" (en Herod, 2011: 1). Seguramente, el análisis de las "escalas" o de los "niveles" de la vida social no constituye el eje de los textos de Pérez-Agote, pero parece claro que su obra ofrece valiosos análisis para aproximarse a su estudio teniendo en cuenta su multiplicidad, su variabilidad, contextualidad, carácter relacional y simbólico y su dimensión territorial y espacial. Se ha señalado, además, cómo este tipo de consideración facilita la comprensión de algunas de las dislocaciones modernas, agrandadas por la mundialización, como la existente "entre la dimensión simbólicaterritorial en la que el hombre vive, siente y entiende y la génesis de una nueva realidad y estructura que no tienen sentido para él" (Pérez-Agote, 1996:14). Algo que también sucede con el llamado "salto de escalas", término que el autor no emplea, pero que cabría aplicar sin dificultad a muchas de las situaciones que escudriña.

Es cierto, sin embargo, que quedan otros muchos aspectos por debatir, como la equiparación que se recoge en su obra, en algún caso, de la mundialización con una "realidad desterritorializada", como si los sentimientos de pertenencia o de identidad colectiva tuvieran muchas más dificultades en recrearse una vez sobrepasadas las "totalidades sociales nacionales" (Ibid.: 26), que ofrecerían, "por el momento, al menos, los límites de una significatividad social eficiente" (Íbidem). El distanciamiento entre el ámbito del sentido y el de "las decisiones 
lejanas [que] se imponen" (Íbidem), a partir de una escala, parece inspirarse aquí en un cierto esquema hiperglobalista o dicotómico, más cercano al planteamiento sobre el hiperespacio de Jameson, o a la brecha creciente entre el espacio de los flujos, o del capital y del poder, de una parte, y los espacios de vida, de otra (Barañano, 2005). Pero hay que recordar también las referencias, presentes también en su obra, a la ciudadanía performativa global, o a los nuevos movimientos sociales de base local, que, sin embargo, al mismo tiempo, tienen un alcance mundial, como el $15 \mathrm{M}$, ejemplos ambos que ilustran la idea de la reconfiguración multiescalar de lo social hoy.

Para acabar estas páginas, cabe referirse, muy sucintamente, a una cuestión enunciada al inicio texto, y no por ello menos importante: la relativa a los debates sobre el estatuto de la noción de escala, y los paralelismos de dichos debates con algunos de los abordados en la obra de Pérez-Agote en relación con los conceptos empleados, como el nacionalismo o la nación. Al respecto, cabe sugerir que los debates sobre el estatuto ontológico o epistemológico de la noción de escala guardan cierta semejanza con los que se recogen en la obra de Alfonso PérezAgote respecto de las nociones de nación o de nacionalismo vasco. El autor las conceptualiza como categorías sociales, construidas por la propia sociedad, más que como nociones sociológicas. La rotunda afirmación de Pérez-Agote de que "la nación no es un concepto científico" (Pérez-Agote, 1999: 21), ofrece un buen ejemplo de este debate, reproducido en términos semejantes en el ámbito de la literatura sobre escalas. Frente a la aproximación de Cox o de otros autores, para los cuales la escala "existe como una categoría ontológica", Jones subraya, sobre todo, su condición de herramienta analítica (Cox, 1998a, 1998 b; Jones, 1998; Herod, 2011; Herod y Wright, 2002a, 2002b), si bien su uso tendría consecuencias materiales (Jones, 1998). La discusión sobre el estatuto fundamentalmente ontológico, o bien epistemológico, de estas nociones, y las ambigüedades que las circundan, queda así abierta, de forma paralela, tanto en dicha literatura, como en la obra de Pérez-Agote, algo a lo que, en todo caso, habría que dedicar otro trabajo.

\section{BibLIOGRAFÍA}

Albrow, M., 1997, The Global Age. State and society beyond modernity, Stanford University Press, Stanford.

Anderson, B., 1993, Comunidades imaginadas: Reflexiones sobre el origen y la difusión del nacionalismo, FCE, México. 
Barañano, M., 2005, “Escalas, des/re-anclajes y transnacionalismo. Complejidades de la relación global-local", en A. Ariño (Ed.), Encrucijadas de la diversidad cultural, CIS, Madrid, pp. 425-451.

Beck, U., 1998, ¿Qué es la globalización? Falacias del globalismo, respuestas a la globalización, Paidós, Barcelona.

Beck, U., 2006, Cosmopolitan vision, Polity Press, Cambridge.

Brenner, N., 2001, "The limits to scale?. Methodological reflections on scalar structuration", en Progress in Human Geography, 25, 4, pp. 591-614.

Castells, M., 1997, La sociedad red, 1. La era de la información. Economía, sociedad y cultura, Alianza, Madrid.

Castells, M., 2006, la sociedad red: una visión global, Alianza, Madrid.

Cox, K. R., 1998a, "Representation and power in the politics of scale", en Political Geography, vol. 17, n 1, pp. 41-44.

Cox, R. K., 1998b, "Spaces of dependence, spaces of engagement and the politics of scale or: looking for local politics", en Political Geography, 17, pp. 1-23.

Delaney D., Leitner, H., 1997, "The political construction of scale", en Political Geography, 16, 2, pp. 93-97.

Giddens, A., 1993, Consecuencias de la modernidad, Alianza, Madrid.

Herod, A., 2011, Scale, Routledge, Londres.

Herod, A., Wright, M. W, 2002a, "Introduction: Theorizing scale", en Geographies of Power: Placing Scale, 10, 22, pp. 1-14.

Herod, A., Wright, M. W., 2002b, "Introduction: Rhetorics of scale", en Geographies of Power: Placing Scale, pp. 147-153.

Jameson, F., 1991, Postmodernism or the cultural logic of capitalism, Verso, Londres.

Jones, K. T., 1998, "Scale as epistemology", en Political Geography, vol. 17, $n^{\circ} 1$, pp. 25-28.

Pérez-Agote, A., 1989, La sociedad y lo social. Ensayos de sociología, Universidad del País Vasco, Bilbao.

Pérez-Agote, A., 1996, "La sociedad se difumina, el individuo se disgrega. Sobre la necesidad de historizar nuestras categorías", en A. PérezAgote e I. Sánchez de la Yncera (Eds.), Complejidad y teoría social, CIS, Madrid, pp. 11-32. 
Pérez-Agote, A., 1999, "Theses sur l'arbitraire de l'être collectif national", en W. Dressler, G. Gatti y A. Pérez-Agote (Eds.), Les nouveaux répères de l'identité collective en Europe, L'Harmattan, Paris, pp. 1932.

Pérez-Agote, A., 2005, "En la era global: ruptura de ecuaciones y dicotomías de la modernidad", en A. Ariño (Ed.), Encrucijadas de la diversidad cultural, CIS, Madrid, pp. 313-336.

Pérez-Agote, A., 2008, Las raíces sociales del nacionalismo vasco, CIS, Madrid.

Pérez-Agote, A., Tejerina, B., Barañano, M., (Eds.), 2010, Barrios multiculturales. Relaciones interétnicas en los barrios de San Francisco (Bilbao) y Embajadores/Lavapiés (Madrid), Trotta, Madrid.

Sassen, S., 2008, Territorio, autoridad, derechos, Katz, Madrid.

Smith, N., 1990, Uneven development: Nature, capital and the production of space, Blackwell, Oxford.

Swyndegouw, E., 1992, "The Mammon quest: 'Glocalization' interspatial competition and the monetary order: The construction of new scales", en M. Dunford y G. Kafkalas (Eds.), Cities and regions in the New Europe: The global/local interplay and spatial development strategies, Belhaven Press, Londres, pp. 39-67.

Swyndegouw, E. 1997, "Neither global not local: 'Glocalization'and the politics of scale", en K. R. Cox (Ed.), Spaces of globalization, Nueva York, The Gilford Press, pp. 137-165.

Wimmer, A., Glick Schiller, N., 2002, "Methodological nationalism and beyond: nation-state building, migration and the social sciences", en Global Networks, vol. 2, 4, pp. 301-334. 